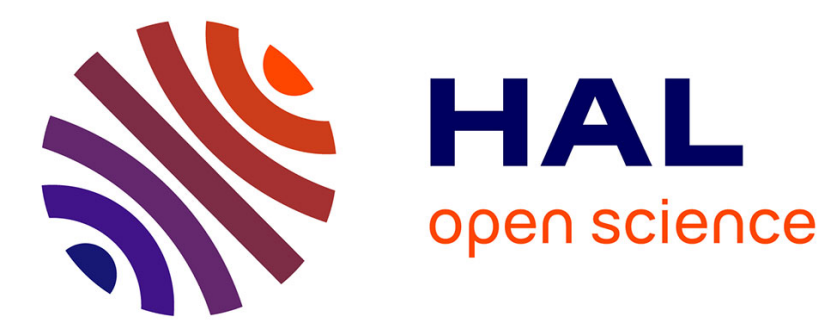

\title{
Cooperative Closed-loop MIMO Selective Transmissions in a HV Environment
}

Olufemi James Oyedapo, Baptiste Vrigneau, Rodolphe Vauzelle

\section{To cite this version:}

Olufemi James Oyedapo, Baptiste Vrigneau, Rodolphe Vauzelle. Cooperative Closed-loop MIMO Selective Transmissions in a HV Environment. SmartGridComm, Nov 2014, Venice, Italy. hal$01277452 \mathrm{v} 2$

\section{HAL Id: hal-01277452 \\ https://inria.hal.science/hal-01277452v2}

Submitted on 16 Dec 2014

HAL is a multi-disciplinary open access archive for the deposit and dissemination of scientific research documents, whether they are published or not. The documents may come from teaching and research institutions in France or abroad, or from public or private research centers.
L'archive ouverte pluridisciplinaire HAL, est destinée au dépôt et à la diffusion de documents scientifiques de niveau recherche, publiés ou non, émanant des établissements d'enseignement et de recherche français ou étrangers, des laboratoires publics ou privés. 


\title{
Cooperative Closed-loop MIMO Selective Transmissions in a HV Environment
}

\author{
O.J. Oyedapo ${ }^{1}$, B. Vrigneau ${ }^{2}$, and R. Vauzelle ${ }^{1}$ \\ ${ }^{1}$ University of Poitiers, XLIM-SIC UMR-CNRS 7252 SP2MI, Téléport 2, \\ Bvd Marie et Pierre Curie, BP 30179, 86962 Futuroscope, France \\ Email $^{1}$ : $\{$ firstname.lastname $\} @$ univ-poitiers.fr \\ ${ }^{2}$ University of Rennes1, CAIRN INRIA-IRISA UMR CNRS 6074, \\ 6 rue de Kerampont, 22300 Lannion, France \\ Email $^{2}$ :baptiste.vrigneau@irisa.fr
}

\begin{abstract}
Integrated communications with sensing and measurements are relevant technologies identified to provide automation for the smart grid (SG) applications. Incorporating intelligence into the electrical grid necessitates the gathering of relevant information in in realtime from variety of electrical utility sources located either at the bulk generation, transmission, distribution or customer domain. The deployment of wireless sensor network (WSN) is an attractive solution for such task, due to certain merits. In this paper, we show how large WSN cluster could benefit from distributed $\max -d_{\min }$ MIMO precoder with a proposed node selection technique offering a realistic implementation. We exploit spatial diversity of the nodes to make the channel state information (CSI) available at transmitting nodes organized into a cluster. Performance evaluation of the nodes selection technique shows substantial amount of energy can be saved in high voltage (HV) power substation channel environment that is corrupted by Additive White Gaussian Noise (AWGN), impulsive noise, and node synchronization errors.

Index Terms-Closed-loop MIMO, cooperation, energy consumption, high voltage substation.
\end{abstract}

\section{INTRODUCTION}

One of the challenges facing electrical utilities is to continuously provide electric power to the consumers reliably without interruption. Automating the power grid will add intelligence to the grid system, hence the term smart grid (SG). SG is expected to optimize the delivery system of electric power by automatic monitoring and protection of interactive and integrated elements located in the grid network especially, the high-voltage (HV) substation networks even at the consumer end [1]. Electric system automation requires the establishment of reliable and self-recoverable system capable of responding quickly to real-time and physical events such that appropriate interventions can be taken if the need be, thereby ensuring constant supply of power without interruption [2]. Such automation may require constant monitoring in real-time, thus necessitating information measurements from various sources. The wireless sen- sor networks (WSN) is an attractive option due to its low cost, ease of deployment, self-organisabilty, self-configurabiliy, e.t.c. Despite these merits, certain challenges posed by WSN with respect to channel of communication include dynamic environment of operation, dynamic WSN topology, issues related to the quality of service (QoS), and especially the limited power sources of the nodes. WSN could implement cooperative Multiple-Input Multiple-Output (MIMO) in order to benefit from performance of classical MIMO transmission technique. By exploiting spatial diversity of nodes, and appropriating cooperation technique, nodes can cooperatively transmit via other nodes (as relays) to the receiver. Cooperation could be fixed, dynamic [3], in which relays transmit the source data in opportunistic manner, cluster-based [3], [4] and/or parallel relays [5] where relays transmit simultaneously to the receiver. In more complex scenarios clusters do cooperate in multihops to transmit and receive data from/to other cluster.

The exploitation of the channel state information (CSI) at the transmitting side (CSI-T) [6] to improve MIMO system's performances in terms of spectral efficiencies or probability of error is well studied. In [7], the Alamouti code antenna selection which requires 2-transmit- and 2-receive-antenna diversity from 3 or 4 transmit antennas, using the orthogonal space time block code (OSTBC) were exploited in terms of energy consumption of nodes. It has been shown that cooperative transmission with multiple antenna is more energy efficient than Single-Input Single-Output (SISO) and multiple hop techniques over long distance [4], [7]. However, short-range transmission cannot benefit from MIMO transmissions, though it outperforms SISO in terms of bit error rate (BER). By exploiting CSI$\mathrm{T}$, precoder-based MIMO transmissions can optimize certain criterion [8], [9], [10] to further improve MIMO system performance. The $\max -d_{\min }$ precoder [11] op- 
timizes the Euclidean distance to reduce the BER of the received data, and has been shown to be more energy efficient compared to open-loop techniques like the OSTBC and the Alamouti code [12]. For this reason, our goal is to further enhance the performance of the closed-loop precoding for the impulsive noise modeled as Middleton noise and peculiar to the $\mathrm{HV}$ power substation.

The $\max -d_{\min }$ precoder implemented in this paper utilizes the closed-loop MIMO with full CSI (FCSI) and the limited CSI (QCSI) for the evaluation of the considered network scenario shown in figure 1 . We have assumed the availability of limited knowledge of channel information for the case of QCSI, in which limited number of bits are fed back to the transmitting nodes by the receiver. This is an important assumption for the implementation of the realistic WSN. Specifically, a very low feedback link is considered, typically 3 to 7 bits, [8], with assumptions that the feedback link is error-free with negligible delays. The aim of this paper is to optimize the energy of transmit nodes via distributed MIMO precoding for communication channel found in HV power substation, implemented with statistical Rayleigh channel and RaPSor ray-tracing tool. A Maximum Likelihood (ML) receiver optimized for the impulsive noise (ML-M) is implemented and compared to the classical Gaussian-based receiver (ML-G). We evaluate the BER performances of ML-G and ML-M based receivers with transmit nodes de-synchronization, and energy consumption using a modified model for our cooperative MIMO.

The contribution of this paper follows:

1) To propose a node selection technique of the max- $d_{\min }$ distributed precoded which exploits spatial diversity of a cluster, i.e. permits to extend the closed loop technique to more than four nodes.

2) Evaluate the performance of the node selection technique in terms of BER and energy cost parameters in HV substation environment characterized by impulsive noise and transmit nodes de-synchronization.

This paper is organized as follows. Section II presents the realistic environmental scenario considered for our cooperative MIMO transmissions. The model and description of $\max -d_{\min }$ precoder based on optimization of the minimum Euclidean distance; and the proposed node selection technique are provided in section III. Section IV presents the performance evaluation of our proposed node selection technique in a Rayleigh channel and RaPSor-generated channel corrupted by AWGN and impulsive noise, with node synchronization errors. Subsequently, energy consumptions of nodes in Joules per bit (J/bit), of the proposed transmission scenarios are evaluated in section V. We conclude this paper in section VI.

\section{THE PROPOSED SCENARIO OF A WSN IN A POWER SUBSTATION}

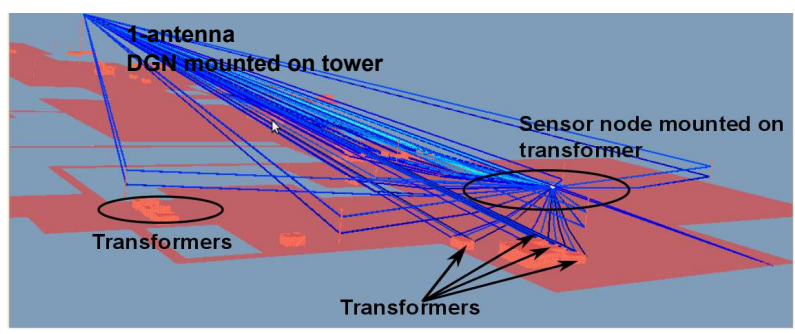

Fig. 1. The Laurentides HV substation with sensor(s) and DGN positions showing interaction of rays with objects in substation - this scenario shows one sensor at a time as permitted by RaPSor.

The location of interest is the Laurentides HV power substation in Canada with the area dimension of $1300 \mathrm{~m} \times 800 \mathrm{~m}$. Without giving exhaustive listing, the equipments and devices located in this substation include circuit-breakers, current transformers, main transformers, and disconnect switches. Additional structures present are concrete walls, buildings, and pylons. In order to model the realistic substation radio channel, a three-dimensional (3D) model of the substation, comprising the equipments and structures previously mentioned were supplied to the RaPSor modeling tool [13]. RaPSor is a ray propagation simulator developed by the XLIM-SIC laboratory of the University of Poitiers. It is open and can be extended based of Netbeans client platform. This simulation tool uses geometric theory of diffraction (GTD) to calculate several paths between a transmitter and a chosen receiver (cf. figure 1). The goal of using this tool is to obtain the complex channel Impulse Response (IR) of each coefficient of the channel matrix $\mathbf{H}\left[n_{r} \times n_{t}\right]$.

In the considered transmission scenario, distributed wireless temperature sensors are deployed and positioned on transformers to form a 10-node cluster. Four Data Gathering Nodes (DGNs) comprising one antenna each are individually placed on a $60 \mathrm{~m}$ high lighting pole. Each transmit node is equipped with a single antenna and it is energy autonomous, though the DGN is a multiantenna node but the energy is not constrained. The proposed solution allows nodes to cooperate in order to transmit information to a DGN mounted on a high point. Eventually, the multi-antenna DGN can be separated into several single antennas mounted on different lighting poles ${ }^{1}$ in order to consider our network as a perfect MIMO system.

\footnotetext{
${ }^{1}$ for RaPSor simulation purpose
} 
III. MIMO TRANSMISSION TECHNIQUE: $\max -d_{\min }$ PRECODING

A. $\max -d_{\min }$ Precoder

For a linear precoder based MIMO system with $n_{r}$ receiving and $n_{t}$ transmitting antennas, the received vector of the max $-d_{\min }$ precoder for maximum likelihood (ML) detection is:

$$
\mathbf{y}=\mathbf{G}_{d} \mathbf{H}_{v} \mathbf{F}_{d} \mathbf{s}+\mathbf{G}_{d} \mathbf{n}_{v}
$$

The CSI is made available at the receiver and the transmitter with $b$ independent data streams such that $b \leq \min \left(n_{t}, n_{r}\right)$, then by singular value decomposition (SVD) of the channel matrix $\mathbf{H}, \mathbf{F}_{v}$ and $\mathbf{G}_{v}$ can be obtained, where $\mathbf{H}_{v}=\mathbf{G}_{v} \mathbf{H F}_{v}=\operatorname{diag}\left(\sigma_{1}, \ldots, \sigma_{b}\right)$, and $\mathbf{H}_{v}$ is the virtual channel matrix, whose elements represent the sub channel gains arranged in descending order. The precoding and decoding matrix can be written as $\mathbf{F}=\mathbf{F}_{v} \mathbf{F}_{d}$ and $\mathbf{G}=\mathbf{G}_{d} \mathbf{G}_{v}$ respectively. Solution of $\max -d_{\min }$ is to find the coefficient of matrix $\mathbf{F}_{d}$ which maximizes the minimum Euclidean distance $d_{\min }$ between the signal points of the received constellation:

$$
\mathbf{F}_{d}=\arg \max _{\mathbf{U}} d_{\min }(\mathbf{U}), d_{\min }(\mathbf{U})=\min _{\mathbf{e} \in \mathfrak{C}^{b}}\left\|\mathbf{H}_{v} \mathbf{U} \mathbf{e}\right\|
$$

where $\mathbf{e}=\left(\mathbf{x}_{k}-\mathbf{x}_{l}\right), k \neq l$, under the mean available transmit power constraint $\operatorname{trace}\left(\mathbf{F}_{d} \mathbf{F}_{d}^{*}\right)=P_{0}$. A promising solution of equation (2) is possible [11] for $b=2$ and a 4-QAM. These solutions are SNR independent but depend on the channel angle $\gamma=\arctan \frac{\sigma_{2}}{\sigma_{1}}$. The solutions for 16-QAM [14] and a suboptimal extension [15] are available. Solutions are not rewritten here due to space restriction.

\section{B. Node selection technique}

Our considered network consists $\mathbb{N}$ set of nodes $N_{g}$ $(g=1,2, \ldots a)$ which, is a superset of two sets of nodes $\mathbb{N}_{1}$ and $\mathbb{N}_{2}$, such that $\left\{\mathbb{N}_{1}, \mathbb{N}_{2}\right\} \in \mathbb{N}$. Any node $s_{i}$ in $\mathbb{N}_{1}$ is a sensor node with potential to become a clusterhead (where $i=1,2, \ldots b$ ). The node $s_{i}$ could cooperate with $n_{c l}-1$ nodes inside a cluster $k$, where $n_{c l}$ is the number of nodes in $k$, and $n_{c l}<a$. Node $D_{h}$ in $\mathbb{N}_{2}$ is a receiver, and a data gathering node (DGN) having relatively higher processing capabilities (where $h=1,2, \ldots c$, and $c<n_{c l}$ ). We propose a node selection algorithm according to the scenario depicted in figure 1, where a cluster $k$ of $n_{c l}$ nodes, placed on power transformers is formed. A node $s_{i}$ having data to transmit declares itself as the clusterhead by implementing the first declaration wins rule - a passive clustering algorithm in [16]. Such node $s_{i}$ then cooperates with $n_{c l}-1$ nodes to transmit its data to $D_{g}$.

In the next step, $n_{c l}$ nodes in cluster $k$ send their training frames $F_{t r a}$ (i.e. $p . n_{c l}$ ) to $D_{g}$ which, then estimates the CSI, and sends back $F_{f b k}$ frame to $n_{t}$ selected nodes in cluster $k$. A $D_{g}$ computes the best $n_{t}$ nodes to transmit utilizing the CSI estimates according to the optimization criteria in (2) from all possibilities, $\mathrm{C}_{n_{t}}^{n_{c l}}=\frac{n_{c l} !}{n_{t} !\left(n_{c l}-n_{t}\right) !}$. The node selection algorithm is described in algorithm 1 .

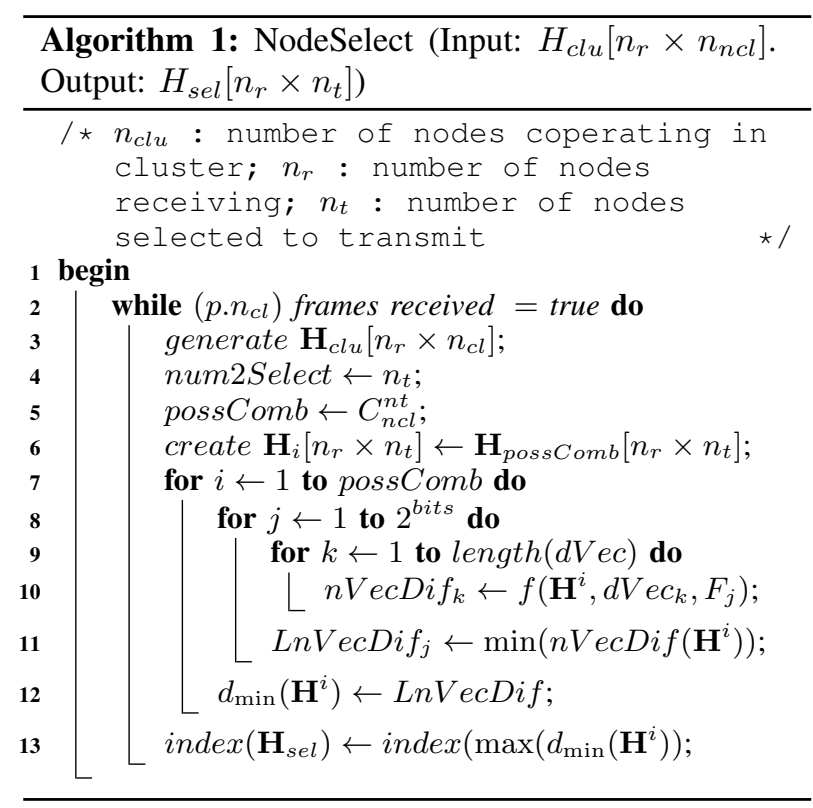

\section{Performance eVAluation - AWGN And IMPULSIVE NOISE}

Using Monte Carlo simulations, we simulate the MIMO channel $\mathbf{H}$ with 10 transmit and 4 receive diversities using $b=2$ independent data streams. For the Rayleigh channel, the entries of $\mathbf{H}$ are independent and identically distributed (i.i.d.) complex Gaussian random variables with mean-zero and variance one. The simulated AWGN vector elements are zero-mean i.i.d. complex Gaussian with variance $\sigma^{2}$. We obtain matrix, $\mathbf{H}$ of the RaPSor channel as described in section II. At each SNR, $10^{6}$ random $\mathbf{H}$ are generated and transmitted symbol vectors are precoded using the max- $d_{\min }$ precoder with perfect CSI (FCSI) at the sensor nodes and the DGN. We assumed the ML receiver for 4-QAM modulation only.

\section{A. AWGN and Impulsive noise}

The HV substation generates strong radio frequency noise capable of disrupting the operation of communication networks. In particular, noise caused by partial discharge (PD) has attracted more attention in $\mathrm{HV}$ substation [17], and are due to breakdown in dielectric ultimately resulting into impulsive component of current. In order to design communication in such environment, the HV substation environment has to be characterized to accurately estimate communication performances. 


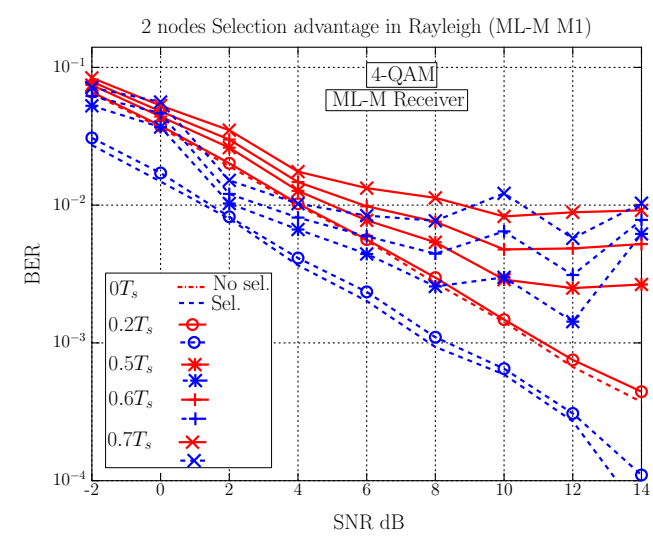

(a) 2 transmit nodes.

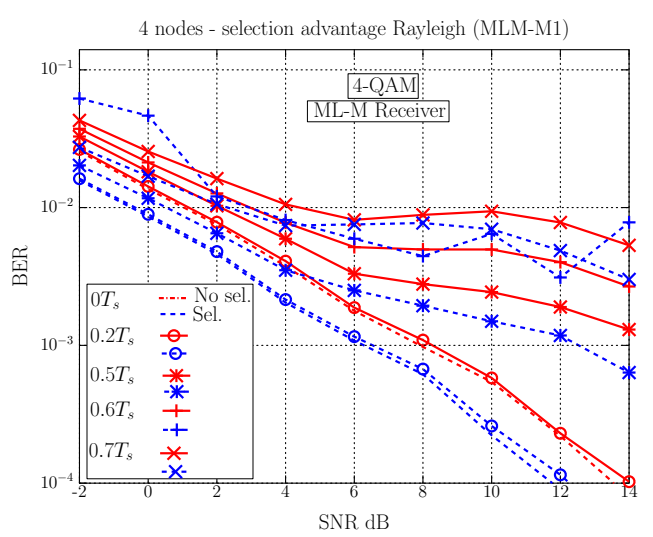

(b) 4 transmit nodes.

Fig. 2. Node selection in Rayleigh faded channel with M1 noise and synchronization errors.

Partial discharge measurements due to high voltage sources were exploited in [12] to determine statistical model of impulsive noise. Having considered various sets of measured noise, they showed that the Middleton Class A statistical model could be used to model the impulsive noise found in HV substation better than the AWGN model. More rigorous study of impulsive noise statistics can be found in [18]. It was shown in [19] that implementing a conventional receiver (ML-G) with the knowledge of AWGN model will experience degraded performances in terms of transmission quality in the presence of impulsive noise. For subsequent sections ML decoders with the knowledge of AWGN and impulsive noise in the channel would be referred to as ML-G and ML-M respectively.

\section{B. Synchronization error}

The presence of synchronization error introduces a superposition of multiple signals at the receiving node. The signals from multiple transmitting nodes are not synchronized with each other and therefore the receiver have no information of the optimal sampling time, because the sampling time of one component of the received signal is not optimal of the others. Desynchronization of the transmitting nodes reduces the energy performance due to super imposition of symbol energy called intersymbol interference (ISI). To evaluate the effect of synchronization error on the performance of our node selection, we implement the model in [20], assuming transmission synchronization error is uniformly distributed in $\left[-\Delta T_{s} / 2,+\Delta T_{s} / 2\right]$ having an error range $\Delta T_{s}$ [20]. We simulate different node synchronization errors ranging from $0.2 T_{s}, 0.5 T_{s}, 0.6 T_{s}$, and $0.7 T_{s}$.

\section{Bit-error-rate}

1) Rayleigh fadding: Since our decoder at the receiver has the knowledge of impulsive noise statistics,
TABLE I

SNR AT TARGET BER OF $10^{-4}$ IN DIFFERENT CHANNELS AND NOISES FOR $\Delta T_{s}=0.2$

\begin{tabular}{|l|l|l|}
\hline Channel \& Noise & SNR with $n_{t}=2$ & SNR with $n_{t}=4$ \\
\hline \hline Rayleigh \& & No Select.: $11.4 \mathrm{~dB}$ & No Select.: $7.0 \mathrm{~dB}$ \\
Gaussian & Select.: $9.6 \mathrm{~dB}$ & Select.: $5.2 \mathrm{~dB}$ \\
\hline Rayleigh \& & No Select.: $16 \mathrm{~dB}$ & No Select.: $14 \mathrm{~dB}$ \\
Impulsive & Select.: $14 \mathrm{~dB}$ & Select.: $12.2 \mathrm{~dB}$ \\
\hline RaPSor \& & No Select.: $18.6 \mathrm{~dB}$ & No Select.: $15.4 \mathrm{~dB}$ \\
Impulsive & Select.: $15.3 \mathrm{~dB}$ & Select.: $14.2 \mathrm{~dB}$ \\
\hline \hline
\end{tabular}

the theoretical ML can be expressed as in [12]. Simulation results for the BER evolutions are shown in figures 2 and 3 for ML-M decoding in Rayleigh faded and RaPSor generated channels respectively. Observe that in figure 2, our node selection technique improves the BER of the received data with significant gains at low synchronization error of $0.2 T_{s}$, even at synchronization error of $0.5 T_{s}$, a slight improvement is noticeable. Selection gains of about $2 \mathrm{~dB}$ and $1.8 \mathrm{~dB}$ are obtained for 2 and 4 selected nodes respectively at $0.2 T_{s}$ synchronization error (cf. figure 2 and table I).

2) RaPSor fadding: Similarly for the RaPSor generated channel, selection gains of $3.3 \mathrm{~dB}$ and $1.8 \mathrm{~dB}$ at $0.2 T_{s}$ synchronization error are obtained for 2 and 4 selected nodes respectively. Observe that the transmission system is quite stable with 2 selected nodes (figure 3(a)), but as the number of selected cooperating nodes reaches 4 (figure 3(a)), our selection algorithm becomes sensitive particularly at $0.5 T_{s}$ synchronization error. One should note that combination of synchronisation error and impulsive noise make increase the BER at around $10 \mathrm{~dB}$.

\section{ENERGY CONSUMPTION}

\section{A. Energy consumption model}

Since we are only interested in the energy of the sensor nodes, we start by expressing the total energy consumption $E_{\text {coop }}$ as in [4], including communication 


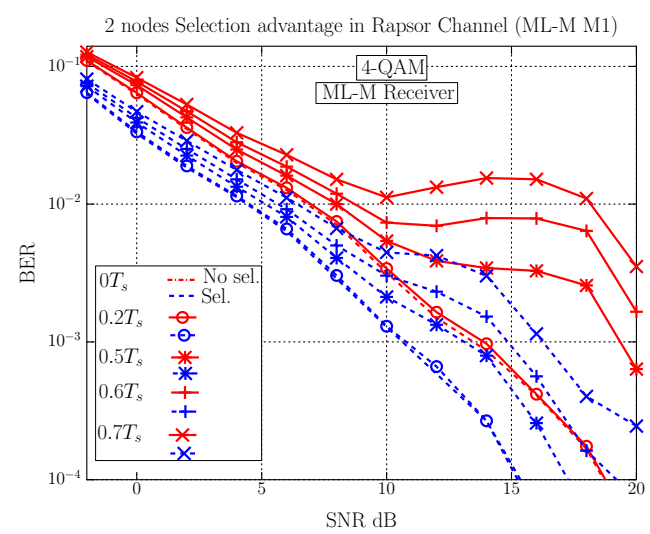

(a) 2 transmit nodes.

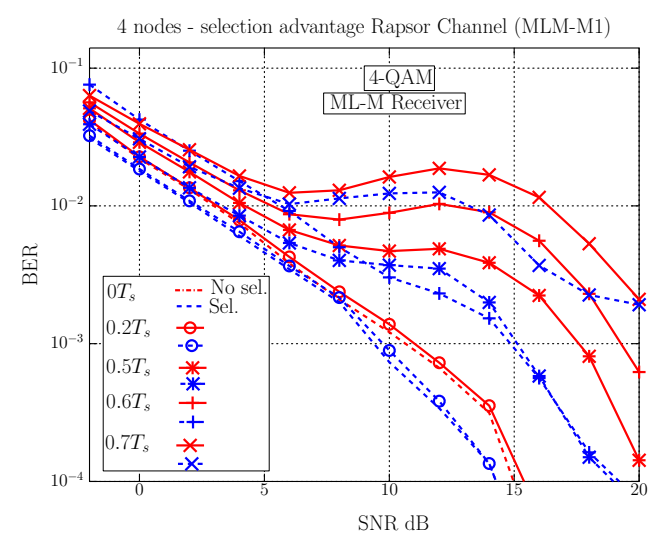

(b) 4 transmit nodes.

Fig. 3. Node selection advantage in RaPSor channel with M1 noise and synchronization errors.

at the cooperation stage:

$$
E_{\text {coop }}=E_{\text {cluster }}+E_{\text {Tra }}+E_{\mathrm{Fbk}}+E_{\text {data }}
$$

where $E_{\text {cluster }}, E_{\mathrm{Tra}}, E_{\mathrm{Fbk}}$, and $E_{\text {data }}$ represent the energy of cluster transmission, the training phase, energy incurred during feedback, and MIMO data packet transmission respectively. The overall power consumption of a RF system can be broadly divided into $(i)$ power consumption due to all power amplifiers $P_{P A}$ and (ii) power consumption due to other circuit blocks $P_{c c t}$. The $P_{P A}$ can be expressed approximately as:

$$
P_{P A}=\frac{\varepsilon}{\eta} P_{\text {out }}=\frac{\varepsilon}{\eta} \frac{E_{b}}{N_{0}} \frac{(4 \pi)^{2} d^{\alpha} M_{l} N_{r}}{G_{t} G_{r} \lambda^{2}} R_{b}
$$

where $E_{b} / N_{0}$ is the average energy per bit to the noise required for a given BER specification, $R_{b}$ is the system bit rate, $G_{t}$ and $G_{r}$ are the transmitter and receiver antenna gains respectively, $\lambda$ is the carrier wavelength, $M_{l}$ is the link margin compensating the hardware process variations and other additive background noise or interference, $N_{r}$ is the power spectral density (PSD) of the total effective noise at the receiver input [4], [21], $d$ is the transmission distance, $\alpha$ is the channel path loss exponent which could usually lie in the range $[2,4]$ for wireless communications channels. All the parameter values in (3) are defined as a function of $P_{c c t}$ and $P_{P A}$.

\section{B. Discussion - Energy Consumption}

The parameters $G_{t}, G_{r}, M_{l}, P_{c c t}^{C_{i T x}}, P_{c c t}^{C_{i R x}}$ are the same as in [4], the central frequency is $2.5 \mathrm{GHz}, \alpha=$ 3.5 , and the packet size of $L=10^{3}$ bits with a target BER of $10^{-4}$ are considered. Using the values of the SNR at target BER in table I and the energy consumption model described above, energy consumption of nodes for evaluating our node selection technique are shown in figure 4 for Rayleigh channel and figure 3 for RapSor generated channel. The energy consumption of nodes are studied at $0.2 T_{s}$ synchronization error only. Figure 4(a) shows the energy consumption of nodes for Gaussian noise in Rayleigh faded channel, indicating energy per bit (in Joules) consumed as a result of the selection gain and diversity gain. The energy consumption of nodes is analyzed using the node selection technique. At MIMO transmission distance of 1000 meters, about $38 \%$ of energy per bit could be saved when 2-nodes are selected (reduced from 0.24 to $0.15 \mathrm{~J} / \mathrm{bit}$ ), compared to about $29.4 \%$ (reduced from 0.17 to $0.12 \mathrm{~J} / \mathrm{bit}$ ) when 4 nodes are selected. Notice the diversity gain of $4.4 \mathrm{~dB}$ (cf. table I) is the highest in Rayleigh channel with Gaussian noise. This yields a mere $0.03 \mathrm{~J} / \mathrm{bit}$ saving in energy for 4 selected nodes as compared to 2 selected nodes taking all transmission complexity into account.

In figure 4(b), the energy consumption of nodes for Middleton type-1 noise in Rayleigh faded channel is shown. Similarly, at the distance of 1000 meters, about $43 \%$ of energy per bit could be saved when 2-nodes are selected (reduced from 0.4 to $0.7 \mathrm{~J} / \mathrm{bit}$ ), compared to about $33 \%$ (reduced from 0.6 to $0.9 \mathrm{~J} / \mathrm{bit}$ ) when 4 nodes are selected. Increasing the number of transmit nodes permits to reduce the BER but the consumption due to additional transmissions for nodes cooperation offset this expected performance. In this case, the node selection with 2 transmit nodes achieves the better trade-off in terms of performance/complexity.

Lastly, figure 4(c) represents the evolution of consumption energy of nodes for the Middleton type- 1 noise in RaPSor generated channel (at $0.2 T_{s}$ synchronization error). About 50\% of energy per bit could be saved when 2-nodes are selected compared to about $26 \%$ when 4 nodes are selected.

\section{CONCLUSION}

In this paper, we have proposed an extension of distributed-precoder-based ( $\left.\max -d_{\min }\right)$ MIMO transmis- 


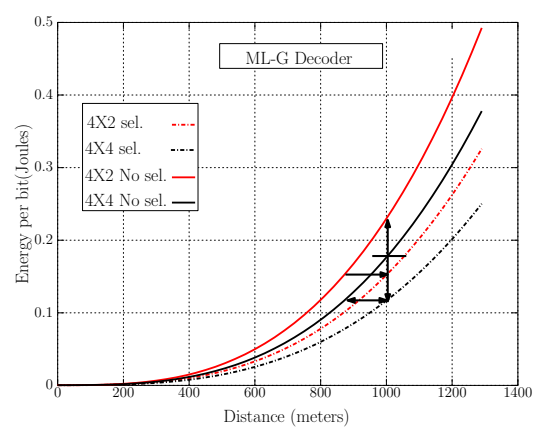

(a) AWGN in Rayleigh channel.

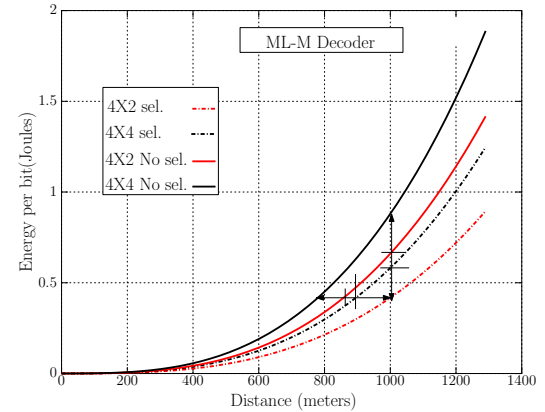

(b) Middleton 1 noise in Rayleigh channel.

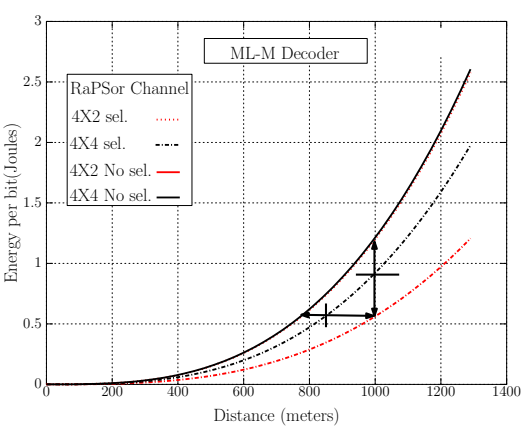

(c) Middleton 1 noise in RaPSor channel.

Fig. 4. Energy consumptions of three different channels (fading and noise) with $\Delta T_{s}=0.2 T_{s}$ synchronization error.

sion with a node selection technique based on the channel CSI in order to take advantage of spatial distribution of nodes. Performance evaluation of this technique, has shown that it is possible for nodes to save substantial energy, especially when the receiver implements a decoder which possesses the knowledge of the channel noise statistics. Our node selection technique performs better for i.i.d channel matrix $\mathbf{H}$ with the Rayleigh fading. We expect the low SNR obtained at the target BER of $10^{-4}$ in all scenarios of 4 selected nodes to yield lower energy per bit but this is offset by additional overhead accrued due to increase in number of nodes (multiplicative factor of 2). Our ultimate aim is to translate this saving in energy to a useful information at the MAC/LLC layer where further energy could be saved by combining more (multiple) parameters for optimized transmission. Generally the simulation results show that our technique is capable of saving more energy at low synchronization error.

\section{REFERENCES}

[1] P. P. Parikh, M. G. Kanabar, and T. S. Sidhu, "Opportunities and challenges of wireless communication technologies for smart grid applications," in IEEE Power and Energy Society General Meeting, 2010, pp. 1-7.

[2] V. C. Gungor and F. C. Lambert, "A survey on communication networks for electric system automation," Journal of Computer Network, vol. 50, no. 7, pp. 877-897, 2006.

[3] A. del Coso, U. Spagnolini, , and C. Ibars, "Cooperative distributed MIMO channels in wireless sensor networks," IEEE J. Sel. Areas Commun., vol. 25, no. 2, pp. 402-414, Feb. 2007.

[4] S. Cui, A. J. Goldsmith, and A. Bahai, "Energy-efficiency of MIMO and cooperative MIMO techniques in sensor networks," IEEE J. Select. Areas Commun., vol. 22, no. 6, Aug. 2004.

[5] R. Madan and N. B. Mehta, "Energy-efficient cooperative relaying over fading channels with simple relay selection," IEEE Trans. Wireless Commun., vol. 7, no. 8, pp. 3013-3025, 2008.

[6] M. Vu and A. Paulraj, "Optimal linear precoders for MIMO wireless correlated channels with nonzero mean in space-time coded system," IEEE Trans. Signal Process., vol. 54, no. 6, pp. 2318-2332, Jun. 2006.

[7] T.-D. Nguyen, O. Berder, and O. Sentieys, "Cooperative MIMO Schemes Optimal Selection for Wireless Sensor Networks," in 65th IEEE VTC, 2007, pp. 85-89.
[8] D. J. Love and J. R. W. Heath, "Limited feedback unitary precoding for spatial multiplexing," IEEE Trans. Inf. Theory, vol. 51, no. 8, pp. 2967-2976, Aug. 2005.

[9] A. Scaglione, S. Barbarossa, G. Giannakis, and H. Sampath, "Optimal Designs for Space-Time Linear Precoders and Decoders,' IEEE Trans. Signal Process., vol. Vol. 50, no. 5, pp. 1051-1064, May 2002.

[10] P. Stoica and G. Ganesan, "Maximum-SNR spatial-temporal formatting design for MIMO channels," IEEE Trans. Signal Process., vol. 50, no. 12, pp. 3036-3042, 2002.

[11] L. Collin, O. Berder, P. Rostaing, and G. Burel, "Optimal minimum distance-based precoder for MIMO spatial multiplexing systems," IEEE Trans. Signal Process., vol. 52, no. 3, pp. 617627, March 2004.

[12] G. Madi, F. Sacuto, B. Vrigneau, B. Agba, Y. Pousset, R. Vauzelle, and F. Gagnon, "Impacts of impulsive noise from partial discharges on wireless systems performance: Application to mimo precoders," EURASIP Journal on Wireless Communications and Networking, Oct. 2011.

[13] Y. Chartois, Y. Pousset, and R. Vauzelle, "MIMO radio channel characterization with a $3 \mathrm{D}$ ray tracing propagation model in urban environment," in European conference on propagation systems ECPS'05, March 2005.

[14] Quoc-tuong, O. Berder, B. Vrigneau, and O. Sentieys, "Minimum distance based precoder for MIMO-OFDM systems using a 16QAM modulation," IEEE ICC, Germany, 2009.

[15] B. Vrigneau, J. Letessier, P. Rostaing, L. Collin, and G. Burel, "Extension of the mimo precoder based on the minimum euclidean distance: A cross-form matrix," IEEE J. Sel. Areas Commun., vol. 2, no. 2, pp. 135-146, April 2008.

[16] M. Gerla, T. Kwon, and P. Guangyu, "On-demand routing in large ad hoc wireless networks with passive clustering," in IEEE WCNC, vol. 1, Sept. 2000, pp. 100-105.

[17] Q. Shan, S. Bhatti, I. A. Glover, R. Atkinson, I. E. Portugues, P. J. Moore, and R. Rutherford, "Characteristics of impulsive noise in electricity substation," in 17th EUSIPCO, August 2009, pp. 2136-2140.

[18] S. V. Vaseghi, Advanced digital signal processing and noise reduction, 4th ed. John Wiley \& sons, 2008, no. ISBN: 978-0470-75406-1.

[19] O. Oyedapo, G. Madi, B. Vrigneau, R. Vauzelle, and N. Richard, "Co-operative closed-loop techniques for optimized data transmission applied to a wsn in a power substation," in $3 r d$ IEEE International Conference on Smart Grid Communications (SmartGridComm), Nov. 2012, pp. 627-697.

[20] T.-D. Nguyen, O. Berder, and O. Sentieys, "Impact of transmission synchronization error and cooperative reception techniques on the performance of cooperative MIMO systems," in IEEE ICC, may 2008, pp. 4601-4605.

[21] G. L. Stüber, Principles of mobile communication. Kluwer Academic Publishers, Second Edition, 2002. 